\title{
BMJ Open TAxonomy of Self-reported Sedentary behaviour Tools (TASST) framework for development, comparison and evaluation of self-report tools: content analysis and systematic review
}

\author{
PM Dall, ${ }^{1}$ EH Coulter, ${ }^{2}$ CF Fitzsimons, ${ }^{3}$ DA Skelton, ${ }^{1}$ SFM Chastin, ${ }^{1}$ \\ on behalf of the Seniors USP Team
}

To cite: Dall PM, Coulter EH, Fitzsimons CF, et al.

TAxonomy of Self-reported Sedentary behaviour Tools (TASST) framework for development, comparison and evaluation of self-report tools: content analysis and systematic review. BMJ Open 2017;7:e13844.

doi:10.1136/bmjopen-2016013844

- Prepublication history for this paper is available online. To view these files please visit the journal online (http://dx.doi.org/10.1136/ bmjopen-2016-013844).

Received 10 August 2016 Revised 30 January 2017 Accepted 3 February 2017

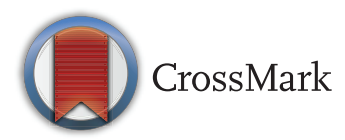

${ }^{1}$ Institute for Applied Health Research, Glasgow Caledonian University, Glasgow, UK

${ }^{2}$ Department of Nursing and Healthcare, Glasgow University, Glasgow, UK

${ }^{3}$ Sport, Physical Education and Health Sciences Institute, Edinburgh University, Edinburgh, UK

Correspondence to Dr P M Dall; philippa.dall@gcu.ac.uk

\section{ABSTRACT}

Objective: Sedentary behaviour (SB) has distinct deleterious health outcomes, yet there is no consensus on best practice for measurement. This study aimed to identify the optimal self-report tool for population surveillance of $\mathrm{SB}$, using a systematic framework.

Design: A framework, TAxonomy of Self-reported Sedentary behaviour Tools (TASST), consisting of four domains (type of assessment, recall period, temporal unit and assessment period), was developed based on a systematic inventory of existing tools. The inventory was achieved through a systematic review of studies reporting $\mathrm{SB}$ and tracing back to the original description. A systematic review of the accuracy and sensitivity to change of these tools was then mapped against TASST domains.

Data sources: Systematic searches were conducted via EBSCO, reference lists and expert opinion.

Eligibility criteria for selecting studies: The inventory included tools measuring SB in adults that could be self-completed at one sitting, and excluded tools measuring SB in specific populations or contexts. The systematic review included studies reporting on the accuracy against an objective measure of SB and/or sensitivity to change of a tool in the inventory.

Results: The systematic review initially identified 32 distinct tools (141 questions), which were used to develop the TASST framework. Twenty-two studies evaluated accuracy and/or sensitivity to change representing only eight taxa. Assessing SB as a sum of behaviours and using a previous day recall were the most promising features of existing tools. Accuracy was poor for all existing tools, with underestimation and overestimation of SB. There was a lack of evidence about sensitivity to change.

Conclusions: Despite the limited evidence, mapping existing SB tools onto the TASST framework has enabled informed recommendations to be made about the most promising features for a surveillance tool, identified aspects on which future research and development of $S B$ surveillance tools should focus.

Trial registration number: International prospective register of systematic reviews (PROPSPERO)/ CRD42014009851.

\section{Strengths and limitations of this study}

- A systematic approach was taken towards classifying self-reported measures of sedentary behaviour, allowing a structured approach to measurement in the future.

- An example of use of the framework is presented, mapping accuracy and sensitivity to change of self-reported sedentary behaviour (SB) measures on to the framework.

- Although designed to be generic, the TAxonomy of Self-reported Sedentary behaviour Tools framework was developed excluding tools measuring $\mathrm{SB}$ in specialised populations and contexts, for example, children or the workplace, and the framework may therefore not cover some aspects of these tools.

- There is the potential for a language bias, as fulltext articles not in English were not included in the systematic reviews.

\section{BACKGROUND}

Physical inactivity is currently at pandemic levels $^{1}$ and is a global public health concern. Sedentary behaviour (SB), an umbrella term for all waking time spent in non-exercising sitting or reclining postures ${ }^{2}{ }^{3}$ such as sitting during work, motorised transport or watching TV, is the largest contributor to inactivity. ${ }^{5}$ Higher levels of SB have been associated with poor physical and mental health, increased risk of chronic disease and less successful ageing. ${ }^{6-9}$ Consequently, several countries, including the UK, have issued recommendations to reduce $\mathrm{SB}$ at all ages as part of their national physical activity guidelines. ${ }^{10}$ Population surveillance is urgently needed to monitor the impact of such policy, track changes in SB over time and to evaluate public health interventions 
targeting SB. In order to provide effective surveillance on which to base future policy decisions, such surveillance tools should be accurate (provide a true measure of the actual amount of SB in a population) and sensitive to change (provide the true difference in SB between two measurement time points). ${ }^{11}$

Objective body-worn sensors, that measure posture, demonstrate good accuracy for measuring total duration of SB against the gold standard of direct observation, ${ }^{12}$ but they are expensive and challenging to use for population surveillance. Self-report tools provide a pragmatic choice for population surveillance and have the potential to provide context-rich information, useful for intervention development. ${ }^{13}$ To date, surveys assessing SB have predominantly used self-report tools, ${ }^{14}$ which are generally adapted from tools not specifically designed to measure that behaviour (eg, tools designed to measure physical activity), ${ }^{15}$ and which have not been evaluated for population surveillance purposes. ${ }^{14}$ No framework currently exists with which to describe and compare SB self-report tools, meaning there is currently no way of systematically selecting an appropriate tool. A previous systematic review of the measurement characteristics of self-report tools measuring SB, reported acceptable to good reliability but low to moderate correlation with a (non-gold standard) criterion measure. ${ }^{13}$ This suggests that self-report measures of $\mathrm{SB}$ are acceptable tools to establish epidemiological evidence of an association between SB and health. ${ }^{13}$ However, it is possible that the scale of the problem may be vastly underestimated, as differences of $2-4$ hours per day $(\sim 20 \%$ of $\mathrm{SB})$ have been reported between self-report and objective tools. ${ }^{16}$

The primary aim of this study was to identify, in a systematic manner, the optimal self-report tool to measure SB for use in population surveillance. Although selfreport SB tools can and will be used in other areas of research, this study focussed on population surveillance as an area that is crucial to the development of public health policy. To fulfil the primary aim, a framework was created to describe the features of self-report tools measuring SB, the TAxonomy of Self-report Sedentary behaviour Tools (TASST). A systematic inventory of existing self-report tools to measure SB was mapped onto TASST, and the measurement characteristics of these tools, focussing on accuracy and sensitivity to change, were evaluated, with explicit reference to the domains of the taxonomy framework.

\section{METHODS}

The study protocol (International prospective register of systematic reviews (PROSPERO) CRD42014009851), was conducted in three phases. In phase 1 an exhaustive inventory of self-report tools to measure SB in adults and older adults was established using a structured search protocol. Phase 2 was the development of a taxonomy based on content analysis of the items and questions in the tools. In phase 3, a systematic literature review of the measurement characteristics of the tools in the inventory was conducted and mapped onto the taxonomy.

\section{Phase 1: systematic inventory of self-report tools}

The aim of the systematic inventory was to compile an exhaustive list of self-report tools which could be used to measure SB in adults ( $\geq 18$ years) and older adults ( $\geq 60$ years). Since the aim was to identify tools and not to identify articles, this stage does not have the same methodology as a systematic literature review. A literature search was conducted in October 2013 (updated November 2016), for articles reporting SB as an outcome measure. From this review, a list of self-report tools which measured SB was compiled. References lists were reviewed and experts consulted to identify any additional tools to include in the inventory. The inventory then was consolidated to amalgamate tools referred to by different names, and to trace back to the original version. Articles which added significant new questions to tools were included as a separate tool. We defined significant new questions to be at least one question which added or changed the type of SB or the time period considered by the tool. Changes in phrasing of the question were not considered sufficient to be considered as a separate tool. Tools used in a single study and those without names/acronyms were included as separate tools.

To be included in the inventory, tools had to: be suitable for use for large-scale population studies of adults or older adults, including being suitable for selfcompletion by the respondent at a single point in time (a pragmatic requirement to minimise participant burden); and measure SB or a proxy measure of SB (eg, TV viewing). Although there is great interest in the SB across many populations and contexts, for pragmatic purposes, initial taxonomy development was limited to a core of self-report tools widely applicable to the general adult population. Therefore, tools were excluded from the inventory: if they were designed specifically to assess SB in children or other specialised populations (eg, medical conditions); if they were designed specifically to assess SB in a specialised context (eg, workplace or care settings); if continuous reporting over extended periods of time required (eg, diaries or time-use surveys) or if significant interviewer interactions were required. Self-report tools that could be administered by telephone or interview were not automatically excluded; however, tools such as the previous day recall (PDR), ${ }^{17}$ in which the interviewer works through lists of several hundred items, were excluded.

\section{Phase 2: development of a taxonomy}

Only tools identified in the initial search were used to develop the taxonomy. The original text was extracted for each question relating to $\mathrm{SB}$ in each of the selfreport tools identified in the inventory. Content analysis was conducted on the text to extract all of the attributes 
in the questions that were used to describe and constrain what aspect of SB was measured by that question. For example, in the question 'During the last 7 days, how much time did you usually spend sitting on a week day?', attributes extracted relating to the measurement of SB would be 'during the last 7 days', 'time spent sitting' and 'on a week day'. Attributes were then grouped into mutually exclusive domains covering similar aspects of measurement, and categories within those domains were defined iteratively. A new category was created each time a tool did not fit within an existing category. The full taxonomy was then assembled and streamlined by merging categories with overlapping meaning. Finally, consideration was given to potential future developments of self-report tools to measure SB, such as the growing interest in the pattern of accumulation of SB, by adding any categories to the taxonomy considered useful in the future. The resulting taxonomy was then tested by ensuring that all tools could be classified similarly by two independent researchers and that the taxonomy fully defined the tool.

\section{Phase 3: systematic review of measurement characteristics}

Finally, a systematic literature search in relevant health databases was conducted in December 2014 (updated November 2016) via EBSCO host. The search combined the name of the tool including variants and acronyms (except where the acronym was also a common word, eg, Past-day Adults Sedentary Time questionnaire (PAST), Measuring Older adults' Sedentary Time questionnaire (MOST)), with search terms relating to measurement characteristics (valid*/reliab*/repons*/sensitiv* /calibrat* /accura* /agreement / psychometric* /clinimetric* /"measurement characteristics" / reliability and validity (Medical Subject Headings $(\mathrm{MeSH}))$ ). Articles were included only if they reported in English on the accuracy of a tool in the inventory against an objective criterion measure of $\mathrm{SB}$, and/or sensitivity to change. Although articles were only included in the review if they assessed accuracy or sensitivity to change, the search terms included a wide range of psychometric properties in order to maximise the chances of finding eligible articles.

Exclusion by title, then abstract, then full text was conducted by two researchers from a pool of five (PMD, EHC, CFF, SFMC and CL). In the case of disagreement, the article was carried forward in to the next round, or at full-text stage a third researcher was consulted to ensure consensus. Data (tool, criterion, population, statistical analysis, accuracy of sedentary behaviour and sensitivity to change of sedentary behaviour) were extracted and quality was assessed independently by two researchers from a pool of three (PMD, CFF, SFMC). Disagreements were resolved by discussion. Quality was assessed using QualSyst, ${ }^{18}$ modified to include an additional item for the criterion measure. As per the QualSyst guidelines, the quality score for the article (range 0-1) was used to identify common methodological strengths and flaws, rather than as an objective representation of high/low quality. Accuracy and sensitivity to change extracted from included articles were reported for tools in relation to the TASST taxonomy.

\section{RESULTS}

\section{Inventory}

The systematic inventory identified 37 distinct self-report tools used to measure SB in adults and older adults, 32 of which were identified in the initial search and used to form the taxonomy (table 1). The International Physical Activity Questionnaire (IPAQ) was originally developed with four different versions, which were included separately in the inventory (combinations of the long and short versions, and last 7 days and usual week recall). The 45 and Up Study asked different questions in its baseline and follow-up questionnaires, which have been included as separate tools. Three tools, termed "modified' versions, were included where questions had been added or modified to the original tool (European Prospective Investigation of Cancer (EPIC)-Norfolk Physical Activity Questionnaire (EPAQ2), National Health and Nutrition Examination Survey (NHANES) and IPAQ-L, representing a 5 th version of the IPAQ in the inventory), and were considered to form a substantially different version. Some tools identified were used in only a single study, and these were included in the inventory, referred to by the study name. The 32 tools in the original inventory comprised of 141 individual questions, consisting of between one and 20 questions per tool. An evaluation of the content of these individual items formed the basis of the TASST taxonomy.

\section{TAxonomy for Self-report Sedentary Behaviour Tools}

The taxonomy derived from the inventory of self-report tools to measure SB (figure 1) comprises of four domains, which characterise different aspects of the tool: type of assessment, recall period, temporal unit and assessment period. All four aspects are required to describe the tool. Within each aspect, the taxonomy functions as a tree, meaning you can identify a single end point (taxon) which fully describes each question in a tool.

The type of assessment domain of the taxonomy covers the way that the outcome of time spent in SB is derived from the tool. Tools can ask about a single aspect of SB (1.1 single item), or a composite aspect (1.2 composite). Tools using a single item of assessment will generate all of their information about SB within the relevant period of assessment in a single question. That single item can either ask about sitting time directly (1.1.1 direct measure) or it can ask about a single behaviour related to SB which is then used as a proxy measure of SB duration (1.1.2 proxy measure). Composite items of assessment ask multiple questions about several aspects of SB for the same period of 
Table 1 Tools measuring SB for population surveillance identified in the inventory

\begin{tabular}{|c|c|c|}
\hline Acronym & Name of tool/study & Key reference \\
\hline $45 U p-B$ & 45 and Up Study, baseline questionnaire & 19 \\
\hline $45 U p-F$ & 45 and Up Study, follow-up questionnaire & 19 \\
\hline ACS2 & American Cancer Society, Cancer Prevention Study cohort II & 20 \\
\hline ALTS & Australian Leisure Time Sitting questionnaire & 21 \\
\hline AusDiab & The Australian Diabetes Obesity and Lifestyle study & 22 \\
\hline $\mathrm{CCHS}$ & Canadian Community Health Survey & 23 \\
\hline CFS & Canadian Fitness Survey & 24 \\
\hline CHAMPS & Community Health Activities Model Program for Seniors physical activity questionnaire & 15 \\
\hline ELSA & English Longitudinal Study of Ageing & 25 \\
\hline EPAQ2 & $\begin{array}{l}\text { European Prospective Investigation of Cancer (EPIC)-Norfolk Physical Activity } \\
\text { Questionnaire }\end{array}$ & 26 \\
\hline mod EQPAQ2 & modified version of the EPIC-Norfolk Physical Activity Questionnaire & 27 \\
\hline GPAQ & Global Physical Activity Questionnaire & 28 \\
\hline HSE & Health Survey for England & 29 \\
\hline HUNT3 & Nord-Trøndelag Health Study 3 & 30 \\
\hline IPAQ-L I7d & International Physical Activity Questionnaire, Long version, last 7 days & 31 \\
\hline IPAQ-L uw & International Physical Activity Questionnaire, Long version, usual week & 31 \\
\hline mod IPAQ-L & modified version of the International Physical Activity Questionnaire, Long version & 32 \\
\hline IPAQ-S I7d & International Physical Activity Questionnaire, Short version, last 7 days & 31 \\
\hline IPAQ-S uw & International Physical Activity Questionnaire, Short version, usual week & 31 \\
\hline LASA & Longitudinal Ageing Study Amsterdam & 33 \\
\hline MLTPAQ & Minnesota Leisure Time Physical Activity Questionnaire & 34 \\
\hline MOST & Measuring Older adults' Sedentary Time questionnaire & 35 \\
\hline NHANES & National Health and Nutrition Examination Survey & 36 \\
\hline mod NHANES & modified version of the National Health and Nutrition Examination Survey & 37 \\
\hline NHS2 & Nurses Health Survey II & 38 \\
\hline $\begin{array}{l}\text { NIH-AARP } \\
\text { DHS }\end{array}$ & $\begin{array}{l}\text { National Institutes of Health-American Association of Retired Persons (NIH-AARP) Diet } \\
\text { and Health Survey }\end{array}$ & 39 \\
\hline NSWPAS & New South Wales Physical Activity Survey & 40 \\
\hline PASE & Physical Activity Scale for the Elderly & 41 \\
\hline PAST & Past-day Adults Sedentary Time questionnaire & 42 \\
\hline PAST-U* & Past-day Adults Sedentary Time questionnaire-University version & 43 \\
\hline PCSpa & prospective cohort study (Spain) & 44 \\
\hline SBQ & Sedentary Behaviour Questionnaire & 45 \\
\hline SHS & Scottish Health Survey & 46 \\
\hline SIT-Q* & SIT-Q & 47 \\
\hline SIT-Q-7d* & past seven day version of the SIT-Q & 48 \\
\hline STAR-Q* & Sedentary Time and Reporting Questionnaire & 49 \\
\hline STAQ $^{*}$ & Sedentary, Transportation and Activity Questionnaire & 50 \\
\hline
\end{tabular}

Acronym: the commonly used acronym of the tool, or the short identifier adopted for this article. Name of tool: the name of the tool, or the name of the single study using these questions/tool. Key reference: references provided here are not exhaustive, but refer either to an early or well-cited description of the tool, or the study in which the tool was used or adapted. Tools marked with an asterisk (*) were identified in the updated search, and were not used to create the taxonomy.

$\mathrm{SB}$, sedentary behaviour.

assessment. One form of composite item would be to ask about the pattern (ie, frequency and timing) of SB accumulated throughout the recall period (1.2.1 pattern). However, the most common form of composite item is created as a sum (1.2.2 sum) of the time spent in $\mathrm{SB}$ in a range of different activities or situations. The sum can be formed from questions asking about specific behaviours (1.2.2.1), activities such as TV viewing, hobbies and talking with friends, or they can be based on domains (1.2.2.2), locations or situations where you can sit, such as at home, for transport and at work.

The recall period is total time over which the respondent is asked to consider their SB when answering the questions. The recall period can be anchored to the present time in which case it refers to a specific length of time prior to now, for example, yesterday (2.1 previous day), last week (2.2 previous week) or a longer period such as the last month or year (2.3 longer). The recall period can also be unanchored (2.4), in which case the respondent is not asked about a specific period but is asked about a general period of time, for example, asking about SB in a typical week.

The temporal unit is the duration within the recall period that a respondent is asked to report their SB for. For example, in the question 'on a typical day last week, how long did you sit?' the recall period is the previous 
Figure 1 TAxonomy of Self-reported Sedentary behaviour Tools (TASST).

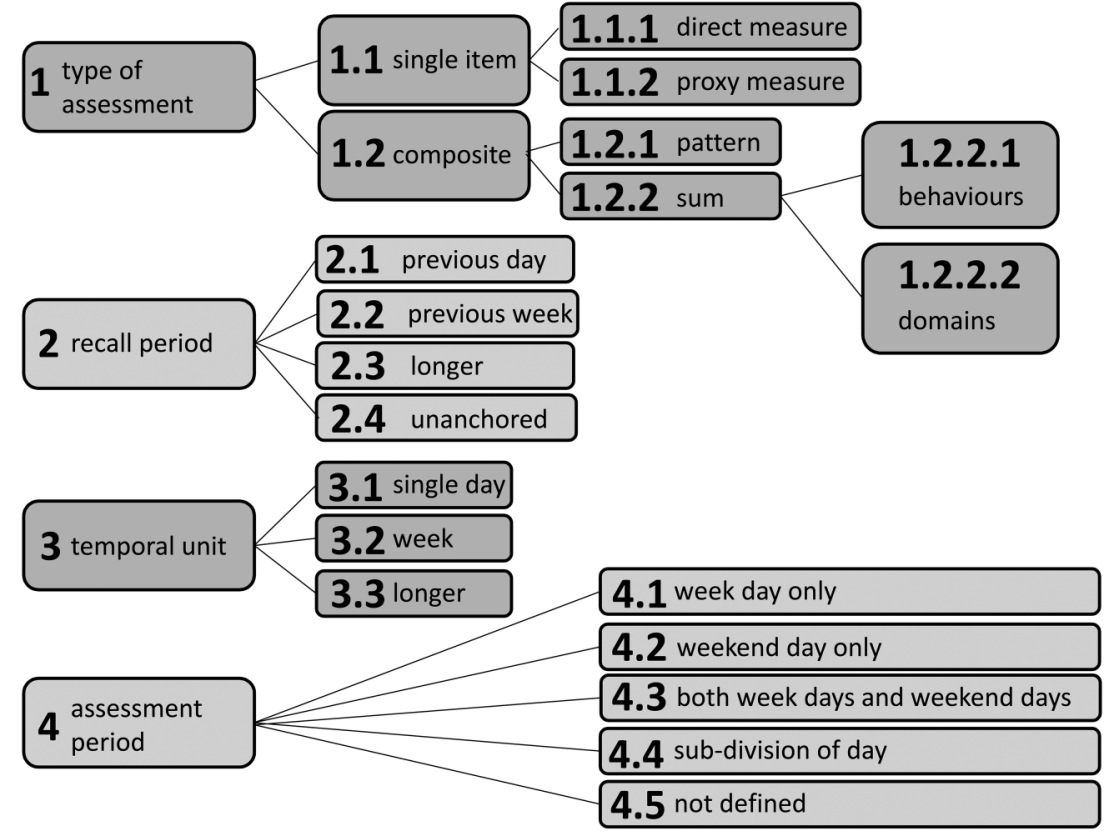

week, but the temporal unit is a day. Within the taxonomy, the temporal units may be a day (3.1), a week (3.2) or longer (3.3). Within a particular recall period, it is possible to have any temporal unit that is of identical or shorter duration than the recall period.

The period of assessment is completed by identifying any specific restrictions that are placed on the type of temporal unit recalled. The categories within the assessment period domain clarify whether a respondent is asked questions regarding a particular type of day, for example, only about week days (4.1), only weekend days (4.2) or is asked about weekdays and weekend days in separate questions (4.3 both). Additionally, the assessment period domain can identify if a respondent is asked about particular subdivisions of the day (4.4) in separate questions, for example, time spent sitting before $6 \mathrm{pm}$. The final taxon in the assessment period is termed 'not defined' (4.5), this represents the situation where a respondent is asked about all temporal units (eg, days) within the recall period (eg, last week) without any specific distinction being made between them. It is a global category, which usually represents a decision not to separate out these categories, as opposed to a failure to define this domain.

\section{Mapping the inventory on to the taxonomy}

The 37 tools identified in the inventory were mapped against the TASST taxonomy (table 2). Approximately half of the tools in the inventory $(n=17)$ used a single item of assessment, 13 used a direct measure and 7 used a proxy measure. Three tools (45 and Up Study, baseline questionnaire (45Up-B), The Australian Diabetes Obesity and Lifestyle study (AusDiab) and National Institutes of Health-American Association of Retired Persons (NIH-AARP) Diet and Health Survey (NIH-AARP DHS)) asked single-item questions about a direct measure and a proxy measure, but not in a manner in which they could be used as a sum, and have therefore been included in the count for both taxa. Proxy measures were predominantly based on TV viewing $(n=5)$. Twenty tools used composite assessment, all of which used a sum as that composite item. The vast majority of sums were formed from questions asking about different behaviours $(n=19)$, with only one sum formed from questions asking about different domains. The tools using a sum of behaviours generally included the common proxy measures of TV viewing $(n=19)$ and computer use $(n=17)$ within the sum. Many tools included questions for behaviours based on leisure pursuits $(n=14)$, in social contexts $(n=9)$, and during transportation $(n=13)$. Often several behaviours of each type were considered in separate questions (eg, asking about time sitting while reading separately from time spent sitting listening to music). Questions based on time working were included in 10 tools, but were explicitly excluded in four tools. Less frequently, tools included questions based on rest $(n=5)$, or used an 'other' category to cover circumstances not explicit within the questions $(\mathrm{n}=7)$.

A little under half of the tools in the inventory used an unanchored recall period $(n=15), 9$ used a previous week recall period and 11 used a longer recall period. Only two tools (PAST, Past-day Adults Sedentary Time questionnaire-University version (PAST-U)) in the inventory used a PDR period. The majority of tools used a temporal unit of a day ( $n=32)$, with five (Australian Leisure Time Sitting questionnaire (ALTS), Canadian Community Health Survey (CCHS), Community Health Activities Model Program for Seniors physical activity questionnaire (CHAMPS), MOST, Nurses Health Survey II (NHS2)) using a temporal unit of a week. A single question within the EPAQ2 questionnaire was based on 


\begin{tabular}{|c|c|c|c|c|c|}
\hline \multicolumn{2}{|c|}{ Taxonomy item } & \multirow[t]{2}{*}{$\mathbf{N}$} & \multirow[t]{2}{*}{ Tools } & \multirow[t]{2}{*}{ Accuracy } & \multirow[t]{2}{*}{$\begin{array}{l}\text { Sensitivity } \\
\text { to change }\end{array}$} \\
\hline 1 & Type of assessment & & & & \\
\hline 1.1 & Single item & 17 & & Underestimate with & + \\
\hline 1.1 .1 & Direct measure & 13 & $\begin{array}{l}\text { 45Up-B; ACS2; AusDiab; CFS; GPAQ; HUNT3; IPAQ-L I7d; IPAQ-L uw; IPAQ-S I7d; } \\
\text { IPAQ-S uw; NIH-AARP DHS; PASE; PCSPa }\end{array}$ & $\begin{array}{l}\text { large systematic and a } \\
\text { random error }\end{array}$ & \\
\hline 1.1 .2 & Proxy measure & 7 & 45Up-B; AusDiab; ELSA; MLTPAQ; NIH-AARP DHS; NSWPAS; SHS & & \\
\hline 1.2 & Composite item & 20 & & Smaller systematic error & + \\
\hline 1.2 .1 & Pattern & 0 & & but there is a potential & \\
\hline 1.2 .2 & Sum & & & to overestimate & \\
\hline 1.2.2.1 & Behaviours & 19 & $\begin{array}{l}\text { 45Up-F; ALTS; CCHS; CHAMPS; EPAQ2; mod EPAQ2; HSE; mod IPAQ-L; LASA; } \\
\text { MOST; NHANES; mod NHANES; PAST; PAST-U; SBQ; SIT-Q; SIT-Q-7d; STAR-Q; } \\
\text { STAQ }\end{array}$ & & \\
\hline 1.2.2.2 & Domains & 1 & NHS2 & & \\
\hline 2 & Recall period & & & & \\
\hline 2.1 & Previous day & 2 & PAST; PAST-U & + & - \\
\hline 2.2 & Previous week & 9 & 45Up-F; ALTS; AusDiab; IPAQ-L 17d; IPAQ-S 17d; mod IPAQ-L; MOST; PASE; SIT-Q-7d & - & + \\
\hline 2.3 & Longer & 11 & $\begin{array}{l}\text { ACS2; CCHS; CHAMPS; EPAQ2; mod EPAQ2; HSE; NHANES; NIH-AARP DHS; SIT-Q; } \\
\text { STAR-Q; STAQ }\end{array}$ & - & - \\
\hline 2.4 & Unanchored & 15 & $\begin{array}{l}\text { 45Up-B; CFS; ELSA; GPAQ; HUNT3; IPAQ-L uw; IPAQ-S uw; LASA; MLTPAQ; mod } \\
\text { NHANES; NHS2; NSWPAS; PCSpa; SBQ; SHS }\end{array}$ & & \\
\hline 3 & Temporal unit & & & & \\
\hline 3.1 & Day & 32 & $\begin{array}{l}\text { 45Up-B; 45Up-F; ACS2; AusDiab; CFS; ELSA; EPAQ2; mod EPAQ2; GPAQ; HSE; } \\
\text { HUNT3; IPAQ-L I7d; IPAQ-L uw; IPAQ-S I7d; IPAQ-S uw; mod IPAQ-L; LASA; MLTPAQ; } \\
\text { NHANES; mod NHANES; NIH-AARP DHS; NSWPAS; PASE; PAST; PAST-U; PCSpa; } \\
\text { SBQ; SHS; SIT-Q; SIT-Q-7d; STAR-Q; STAQ }\end{array}$ & + & + \\
\hline 3.2 & Week & 5 & ALTS; CCHS; CHAMPS; MOST; NHS2 & - & - \\
\hline 3.3 & Longer & 0 & & - & - \\
\hline 4 & Assessment period & & & & \\
\hline 4.1 & Weekdays only & 2 & IPAQ-S I7d; IPAQ-S uw & - & + \\
\hline 4.2 & Weekend days only & 0 & & - & - \\
\hline 4.3 & $\begin{array}{l}\text { Both weekdays and } \\
\text { weekend days }\end{array}$ & 14 & $\begin{array}{l}\text { 45Up-F; AusDiab; ELSA; HSE; IPAQ-L I7d; IPAQ-L uw; mod IPAQ-L; LASA; NSWPAS; } \\
\text { PCSpa; SBQ; SHS; SIT-Q-7d; STAQ }\end{array}$ & + & - \\
\hline 4.4 & Subdivision of the day & 1 & EPAQ2 & + & - \\
\hline 4.5 & Not defined & 21 & $\begin{array}{l}\text { 45Up-B; ACS2; ALTS; CCHS; CFS; CHAMPS; EPAQ2; mod EPAQ2; GPAQ; HUNT3; } \\
\text { MLTPAQ; MOST; NHS2; NHANES; mod NHANES; NIH-AARP DHS; PASE; PAST; } \\
\text { PAST-U; SIT-Q; STAR-Q }\end{array}$ & Better for older adults & + \\
\hline
\end{tabular}

Full names for the acronyms reported in the tools column can be found in table 1. Recommendations in bold are backed by evidence from the systematic review. Recommendations which are not bold are theoretical but no evidence could be found in the literature; ' + ' represents a positive attribute; '-' a negative attribute.

$\mathrm{SB}$, sedentary behaviour. 
a temporal unit longer than a week, but the other three questions in that tool were based on a temporal unit of a day. Just over half the tools $(n=21)$ did not define specific days or time periods in their questions, but asked about the temporal unit within the recall period as a single entity. A total of 16 tools used questions specifically referring to week or weekend days, 14 asking about week and weekend days, while 2 asked only about week days. Only one tool (EPAQ2) referred to specific subdivisions of the day in their questions.

\section{Systematic search for measurement characteristics}

The systematic search returned 7221 references, and after removal of duplicate and assessment against exclusion criteria ( $>99 \%$ agreement between reviewers), a total of 22 studies were included in the review (figure 2, table 3).

\section{Criterion measure}

None of the studies tested the accuracy of the tool against direct observation. Only five studies ${ }^{16} 42434863$ used a postural sensor that actually measures sitting time objectively (activPAL), the other 17 used an accelerometer built to measure low movement as a criterion measure (ActiGraph, actiHeart).

\section{Statistical analysis}

Accuracy and limits of agreement were usually derived from Bland and Altman plots. Sensitivity to change was defined differently in the two articles which reported this measurement characteristic; one used t-test statistics, ${ }^{42}$ one used the Guyatt Index. ${ }^{35}$

\section{Study quality}

Studies which scored highly for quality tended to be purposefully designed to test measurement characteristics, rather than secondary analysis of data collected for another purpose. The most common loss of quality was

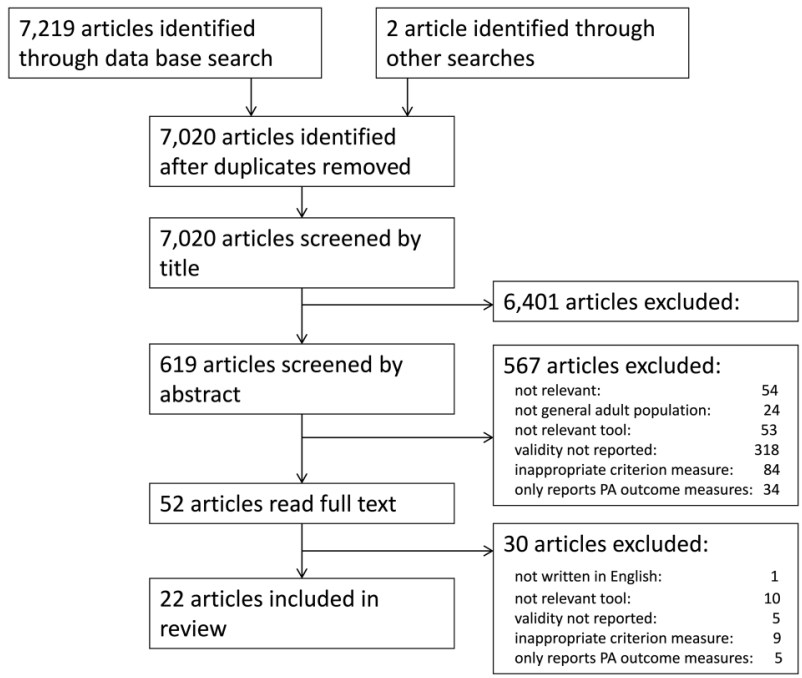

Figure 2 Preferred Reporting Items for Systematic reviews and Meta-Analyses (PRISMA) diagram of the validation systematic review. due to the use of accelerometers which assess low movement (eg, ActiGraph) as a criterion measure, as this does not measure the primary aspect of the definition of SB (ie, posture). Another issue which lowered quality was the manipulation of the criterion measure without clear justification. For example, some studies manipulated the count threshold (used to define SB) or included only SB bouts longer than a particular duration without justification or solid rationale.

\section{Tools and measurement characteristics}

Table 3 summarises the results reported by these studies, arranged per measurement tool and mapped against the relevant taxon. Very few of the existing tools to measure SB using self-report have actually been investigated for these measurement characteristics. Accuracy has been reported for 10 out of the 37 tools identified in the inventory (IPAQ-L 17d, IPAQ-S 17d, Global Physical Activity Questionnaire (GPAQ), MOST, CHAMPS, Longitudinal Ageing Study Amsterdam (LASA), PAST, PAST-U, Sedentary, Transportation and Activity Questionnaire (STAQ), SIT-Q-7d). The most tested tools were the IPAQ in its long form, last 7 days $^{16}{ }^{51-55}$ and short form, last 7 days. ${ }^{5-60}$ The SIT-Q-7d was tested in three studies, ${ }^{48}{ }^{63-64}$ and the CHAMPS was investigated in two studies. ${ }^{15}{ }^{62}$ Information for other tools, GPAQ ${ }^{61}$

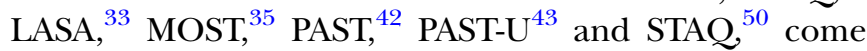
from single studies. Reports of sensitivity to change are only available for two tools, MOST ${ }^{35}$ and PAST. ${ }^{42}$

\section{Taxa tested}

The literature provides measurement characteristics information for eight distinct full taxa:

- 1.1.1/2.2/3.1/4.3 with six studies on IPAQ-L usual week (uw);

- 1.1.1/2.2/3.1/4.1 with five studies on IPAQ-S uw;

- 1.1.1/2.4/3.1/4.5 with one study on GPAQ;

- 1.2.2.1/2.1/3.1/4.5 with one study on PAST and one study on PAST-U;

- 1.2.2.1/2.2/3.1/4.3 with three studies on SIT-Q-7d;

- 1.2.2.1/2.2/3.2/4.5 with one study on MOST;

- 1.2.2.1/2.3/3.2/4.5 with two studies on CHAMPS;

- 1.2.2.1/2.4/3.1/4.3 with one study on LASA and one study on STAQ.

For the assessment type, there is information for direct measures via single item (1.1.1, 12 studies) and for composite sums of behaviours (1.2.2.1, 10 studies). However, there is no information for direct proxy measures (1.1.2). For recall period, there is information on all four possible categories (2.1 previous day, 2 studies; 2.2 previous week, 16 studies; 2.3 longer, 2 studies and 2.4 unanchored, 2 studies). The unanchored recall period (2.4), used by $40 \%$ of the tools in the inventory, is particularly under-represented with only two studies in the validation review. For temporal scale, there is mostly information for assessment at day scale (3.1, 20 studies) and only three studies for the temporal scale of a week (3.2). This is broadly representative of usage by tools in 
Table 3 Measurement characteristics of tools measuring SB, presented by tool and taxon

\begin{tabular}{|c|c|c|c|c|c|c|c|c|}
\hline Tool & $\begin{array}{l}\text { Taxon (refer to } \\
\text { figure 1) }\end{array}$ & $\mathbf{N}$ & $\begin{array}{l}\text { Population } \\
\text { (Country) }\end{array}$ & $\begin{array}{l}\text { Criterion measure } \\
\text { (definition of SB) }\end{array}$ & $\begin{array}{l}\text { QualSyst } \\
\text { Score }\end{array}$ & $\begin{array}{l}\text { Agreement (hours/day tool- } \\
\text { criterion (limit of agreement) }\end{array}$ & $\begin{array}{l}\text { Sensitivity to } \\
\text { change }\end{array}$ & Ref \\
\hline \multirow[t]{6}{*}{$\begin{array}{l}\text { IPAQ-long } \\
\text { I7d }\end{array}$} & \multirow[t]{6}{*}{ 1.1.1/2.2/3.1/4.3 } & 1508 & A \& OA (Greenland) & actiHeart $(<1.5 \mathrm{MET})$ & 0.67 & $\begin{array}{l}-3.0 \text { (not reported) for adults } \\
-6.0 \text { (not reported) for older adults }\end{array}$ & - & 51 \\
\hline & & 542 & A (Netherlands) & Actigraph (<100 count/min) & 0.78 & $-1.6(-6.43 .2)$ & - & 52 \\
\hline & & 980 & A (Sweden) & Actigraph (<100 count/min) & 0.67 & $+2.2(-4.59 .5)$ & - & 53 \\
\hline & & 69 & $A(U K)$ & $\begin{array}{l}\text { activPAL (sitting/lying } \\
\text { postures) }\end{array}$ & 0.78 & $-2.2(-7.23 .7)$ & - & 16 \\
\hline & & 317 & A (Chile) & Actigraph (<100 count/min) & 0.78 & $-1.1(-3.81 .5)$ & - & 54 \\
\hline & & 346 & A \& OA (Switzerland) & Actigraph (<150 count/min) & 0.78 & $-3.8(-9.31 .7)$ & - & 55 \\
\hline \multirow[t]{5}{*}{$\begin{array}{l}\text { IPAQ-short } \\
\text { I7d }\end{array}$} & \multirow[t]{5}{*}{ 1.1.1/2.2/3.1/4.1 } & 1751 & A \& OA (Norway) & Actigraph (<100 count/min) & 0.67 & $\begin{array}{l}-1.8 \text { (not reported) for adults } \\
+3.5 \text { (not reported) for older adults }\end{array}$ & - & 56 \\
\hline & & 144 & A (Nigeria) & Actigraph (<100 count/min) & 0.78 & $-3.0(-8.52 .5)$ & - & 57 \\
\hline & & 54 & OA (Sweden) & Actigraph (<100 count/min) & 0.56 & -1.5 (not reported) & - & 58 \\
\hline & & 127 & OA (USA) & Actigraph (<50 count/min $)$ & 0.72 & $-4.4(-10.0-1.4)$ & - & 59 \\
\hline & & 50 & $A \& O A(U K)$ & Actigraph (<50 count/min) & 0.72 & $-0.5(-1.90 .8)$ & - & 60 \\
\hline GPAQ & $1.1 .1 / 2.4 / 3.1 / 4.5$ & 62 & A (Saudi Arabia) & Actigraph (<100 count/min) & 0.67 & $-3.3(-9.73 .1)$ & - & 61 \\
\hline \multirow[t]{2}{*}{ CHAMPS } & \multirow[t]{2}{*}{ 1.2.2.1/2.3/3.2/4.5 } & 870 & OA (USA) & Actigraph (<100 count/min) & 0.72 & $-6.8(-10.62 .4)$ & - & 15 \\
\hline & & 58 & OA (USA) & Actigraph (<100 count/min) & 0.72 & -5.2 (not reported) & - & 62 \\
\hline LASA & \multirow[t]{2}{*}{$1.2 .2 .1 / 2.4 / 3.1 / 4.3$} & 83 & OA (Netherlands) & Actigraph (<100 count/min) & 0.78 & $\begin{array}{l}+0.2 \text { for } 10 \text { items } \\
-2.1(-7.43 .3) \text { for } 6 \text { items }\end{array}$ & - & 33 \\
\hline STAQ & & 88 & A (France) & Actigraph (<150 count/min) & 0.72 & $-2.4(-6.24 .9)$ & - & 50 \\
\hline PAST & \multirow[t]{2}{*}{$1.2 .2 .1 / 2.1 / 3.1 / 4.5$} & 90 & A (Australia) & $\begin{array}{l}\text { activPAL (sitting/lying } \\
\text { postures) }\end{array}$ & 0.72 & $-1.0(-5.63 .8)$ & $\begin{array}{l}\text { t-test was } \\
\text { inconclusive }\end{array}$ & 42 \\
\hline PAST-U & & 57 & A (Australia) & $\begin{array}{l}\text { activPAL (sitting/lying } \\
\text { postures) }\end{array}$ & 0.78 & $0.1(-3.94 .1)$ & - & 43 \\
\hline \multirow[t]{4}{*}{ SIT-Q-7d } & \multirow[t]{4}{*}{ 1.2.2.1/2.2/3.1/4.3 } & 51 & A (Belgium) & $\begin{array}{l}\text { activPAL (sitting/lying } \\
\text { postures) }\end{array}$ & 0.72 & $1.0(-4.8$ 8.2) for Belgian sample & - & 48 \\
\hline & & 402 & $A(U K)$ & actiHeart $(<1.5 \mathrm{MET})$ & & 0.4 (-6.9 8.6) for UK sample & & \\
\hline & & $33 \& 33$ & A \& OA (Belgium) & $\begin{array}{l}\text { activPAL (sitting/lying } \\
\text { postures) }\end{array}$ & 0.83 & $\begin{array}{l}2.3 \text { (only reported as a \%) } \\
0.3(-8.90 .7) \text { for older adults }\end{array}$ & - & 63 \\
\hline & & 442 & OA (Belgium) & Actigraph (<100 count/min) & 0.83 & $1.36(-6.03 .3)$ & - & 64 \\
\hline MOST & 1.2.2.1/2.2/3.2/4.5 & 48 & OA (Australia) & Actigraph (<100 count/min) & 0.67 & $-3.6(-7.4-0.2)$ & $\begin{array}{l}\text { Guyatt Index } \\
0.39(0.47 \\
\text { for Actigraph) }\end{array}$ & 35 \\
\hline
\end{tabular}

For tool acronyms see table 1.

A, adults; CHAMPS, Community Health Activities Model Program for Seniors physical activity questionnaire GPAQ, Global Physical Activity Questionnaire; IPAQ, International Physical Activity

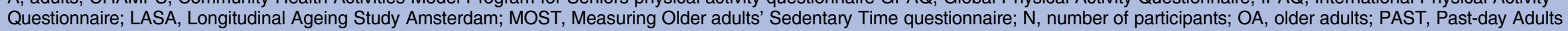
Sedentary Time questionnaire; PAST-U, Past-day Adults Sedentary Time questionnaire-University version; Ref, reference; SB, sedentary behaviour; STAQ, Sedentary, Transportation and Activity Questionnaire; UK, UK; USA, USA of America. 
the inventory. For assessment period, there is information for weekdays only (4.1, five studies) or both weekdays and weekend days (4.3, 11 studies) and for tools with the assessment period not defined (4.5, six studies). The assessment period not defined taxon (4.5), used by over half the tools in the inventory, is under-represented by these validation studies.

\section{Accuracy}

Information for taxon 1.1.1/2.2/3.1/4.3 (IPAQ-L-17d) is not equivocal. The majority of studies reported a large underestimation of total SB time ranging from 1.1 hours in adults ${ }^{54}$ to 6 hours in older adults. ${ }^{51}$ One study reported that tools in this taxon overestimate total SB time by 2.2 hours in adults. ${ }^{53}$ It is clear that the systematic error on estimates of total SB time using tools from this taxon is likely to be very large (several hours/day). The random error is also likely to be very large as the limits of agreement reported were consistently very large. Information for taxon 1.1.1/2.2/3.1/4.1 (IPAQ-S-17d) is a little more consistent for adults. Tools in this taxon seem to underestimate total SB time by 1.5 to 3 hours in adults. However, in older adults this was less clear with reports of underestimation by 4.4 hours $^{59}$ and overestimation by 3.5 hours. ${ }^{56}$ In both populations the error and limits of agreement were large, but not as large as for the previous taxon.

Although not entirely consistent, tools reporting information from a single item as a direct measure of sitting (taxon 1.1.1) tended to underestimate sitting, with underestimation ranging from $-0.5^{60}$ to $-6.0^{51}$ hours per day. Within those tools, the IPAQ-S-17d (reporting only for week days in the past week, taxa 2.2 and 4.1) tended to have better agreement than the IPAQ-L-17d (reporting for both week and weekend days in the past week, taxa 2.2 and 4.3) and the GPAQ (reporting over a longer recall period with the assessment period not defined, taxa 2.4 and 4.5). Tools reporting on a sum of behaviours (taxon 1.2.2.1), were more likely to overestimate sitting than for the single-item direct measure (taxon 1.1.1). Tools which reported on a sum of behaviours over the past day or past week (taxa 1.2.2.1 \& 2.1 or 2.2) tended to have the closest agreement with objective criterion measures with most studies reporting agreement between -1.0 and +2.3 hours per day. Tools which reported sum of behaviours over a longer (taxon 2.3) or unanchored (taxon 2.4) recall period or which had a temporal unit of a week (taxon 3.2) reported larger underestimates (-2.1 to -6.8 hours/day). In particular, the CHAMPS tool, reporting for a recall period of a year (taxon 2.3) with a temporal unit of a week (taxon 3.2), had the largest differences for any tool. However, there were only a few studies reporting on these aspects, and such conclusions are necessarily tentative. Regardless of level of agreement, limits of agreement were large for all tools.

\section{Sensitivity to change}

There is almost no information about sensitivity to change. The two studies that assessed sensitivity to change $^{35} 42$ provided little tangible information. The results were either inconclusive, ${ }^{42}$ or reported the Guyatt index against a criterion measure which does not measure sitting. ${ }^{35}$ While the latter provided some indication that the tools' sensitivity to change was similar to that of an objective measure of low movement, it does not give a clear indication as to whether it is sensitive to a change in total SB time. Neither of these studies reported the minimal detectable change, ${ }^{65}$ a metric which provides an easily interpretable value of the capacity of a tool to detect a change.

\section{DISCUSSION}

A taxonomy (TASST) for the systematic description and comparison of self-reported measures of SB has been established. TASST provides a rigorous framework for informed choice, development and evaluation of selfreport tools. This framework has been used to review the measurement characteristics of existing tools in order to identify the optimum tool for population surveillance. The available evidence about measurement characteristics essential for population surveillance, namely accuracy and responsiveness to change, was insufficient to ascertain which tool currently used in practice is best. Accuracy was poor for all existing tools, with under and overestimation of total time spent in SB and large limits of agreement. In addition, there is a complete lack of evidence about their sensitivity to change. Mapping available evidence onto the TASST framework has enabled informed recommendations to be made about the promising features for a surveillance tool, and identification of the aspects on which future research and development of SB surveillance tools should focus.

The use of a coherent and robust taxonomy (TASST) to systematically evaluate and compare the characteristics of measurement tools is the main strength of this study. However, in terms of accuracy and sensitivity to change, the current published evidence does not cover the entire taxonomy. Consequently, at present, only tentative recommendations can be provided. The taxonomy can be used, however, to identify gaps in current research and provide focussed guidance for future research and development. During the development of TASST, selfreport tools which aimed to measure SB in specific populations (eg, children, those with arthritis) or specialised contexts (eg, workplace) were not considered. However, TASST is a generic framework, so tools specific to these populations may already be fully described by the taxonomy. For example, a question asking about time spent sitting at school which is specific to children, would be covered under the subdivision of the day assessment period (taxon 4.4). Another consequence of the exclusion criteria is that evidence on accuracy and sensitivity to change of tools specific to these populations was not mapped on the taxonomy. Therefore, the conclusions drawn from the measurement characteristics 
in this study are only valid for adults and older adults. Future research should be conducted using the TASST taxonomy to map existing self-report tools covering those populations and contexts currently excluded from taxonomy development (such as children, schools or the workplace) to identify areas for development. In addition, this study has the general limitations common to most systematic reviews, that is, included articles were restricted to those written in English, articles and tools published after the date of search were not included and any relevant articles not identified during the search will have been excluded.

The current study is the first to clearly define and focus on the measurement characteristics required for population surveillance (accuracy and sensitivity to change). There is only one other systematic review reporting on the measurement characteristics of selfreport tools to measure $\mathrm{SB},{ }^{13}$ which concentrated on validity (assessed through rank correlation) and reliability, which are the measurement characteristics relevant to establishing associations between SB and health. In agreement with the previous review, we found that the major flaw of most validation studies was the use of an inadequate criterion measure. The choice of criterion measure depends on the purpose of the tool. While direct observation should be considered the gold standard, if the purpose is to assess total sedentary time, then accurate postural sensors should be adequate (eg, activPAL). In this review, only five out of 22 studies used an adequate criterion measure. Instead, many studies used an accelerometer which measures low levels of movement at the hip (eg, ActiGraph) as a criterion measure, but such tools do not measure SB directly and can misclassify standing as sitting. ${ }^{12}$ Although it is possible that criterion measure may have provided a confounding effect on agreement (eg, tools assessing previous day recall period (taxon 2.1, PAST, PAST-U) were only assessed against the activPAL), no clear trend towards better or worse agreement with a particular type of criterion measure or ActiGraph cut-off was apparent.

Despite the incomplete nature of the evidence, TASST enables the identification of desirable characteristics of self-report tools to measure SB when used for population surveillance. First, tools assessing total SB time as a sum of behaviours (taxon 1.2.2.1; CHAMPS, LASA, MOST, PAST, PAST-U, SIT-Q-7d, STAQ) provided better accuracy than single-item direct measurement (taxon 1.1.1; IPAQ-L-17d, IPAQ-S-17 d and GPAQ) tools, especially when comparing tools with equivalent recall periods. However, this will be dependent on the behaviours or domains included within the sum, and whether they are exhaustive, consistent and mutually exclusive. Tools with a non-exhaustive sum will underestimate total time, for example, the LASA, found that a six-item sum provided a better correlation with SB across the sample, but that a 10 -item sum was more accurate. ${ }^{33}$ Conversely, tools which contain behaviours which might occur concurrently (such as watching TV and using a tablet computer) may lead to an overestimate in total SB time. ${ }^{63}$ Second, tools using a previous day recall period (taxon 2.1, PAST, PAST-U) tended to provide better accuracy than those with longer recall periods (taxa 2.2, 2.3 and 2.4). This corroborates recent research on the validity of computerised survey systems which assess SB using a past-day recall period. ${ }^{17}{ }^{66}$ However, although tools using PDR may be more accurate, it is likely that their sensitivity to change will be less good due to the higher underlying variability in daily SB. ${ }^{67}$

Most tools currently used for population surveillance of SB systematically underestimate the amount of SB by $2-$ 4 hours per day. Yet, self-report tools are still the most practical and economical means of population surveillance. Therefore, policymakers and clinicians should be aware that reports of population SB time are likely to be grossly underestimated, and should be cognisant of this fact when making decisions on implementing, developing and evaluating policy and public health interventions. In addition, policymakers and clinicians should be cautious in interpreting any reported difference in population SB time as a real change. The dearth of information about sensitivity to change of these tools means that we do not know the magnitude of change required to be certain that a change is real and not background variation. Moving forward, development of national and international surveillance systems should not be undertaken assuming that a tool is adequate because it has been used previously. Instead, investment should be made in research to evaluate the sensitivity to change and accuracy of tools to measure SB, paying attention to the potential trade-off between these two measurement characteristics. Such research should be carefully planned, to ensure that meaningful comparisons are investigated. The TASST taxonomy should be used as a useful framework to facilitate such a systematic approach.

Acknowledgements The named authors present the study on behalf of the Seniors - Understanding Sedentary Patterns (Seniors USP) Team, which comprises: DAS (PI), SFMC, Simon Cox, EHC, Iva Čukić, PMD, Ian Deary, Geoff Der, Manon Dontje, CFF, Catharine Gale, Jason Gill, Malcolm Granat, Cindy Gray, Carolyn Greig, Elaine Hindle, Karen Laird, Gillian Mead, Nanette Mutrie, Victoria Palmer, Ratko Radakovic, Naveed Sattar, Richard Shaw, John Starr, Sally Stewart, Sally Wyke. The authors would like to thank Calum Leask (CL) for his help in updating both of the searches in November 2016.

Collaborators Seniors USP Team, which comprises: DAS (PI), SFMC, Simon Cox, EHC, Iva Čukić, PMD, Ian Deary, Geoff Der, Manon Dontje, CFF, Catharine Gale, Jason Gill, Malcolm Granat, Cindy Gray, Carolyn Greig, Elaine Hindle, Karen Laird, Gillian Mead, Nanette Mutrie, Victoria Palmer, Ratko Radakovic, Naveed Sattar, Richard Shaw, John Starr, Sally Stewart, Sally Wyke.

Contributors SFMC and DAS conceived and supervised the study. PMD and SC designed the study. PMD, EHC, CFF and SFMC collected the data and performed the systematic review. PMD, CFF and SFMC analysed the data for the taxonomy. PMD and SFMC analysed the data from the systematic review. All authors interpreted the data and critically revised the manuscript for important intellectual content.

Funding The study was performed in preparation for the Seniors USP: Understanding Sedentary Patterns project, which was supported by the Medical Research Council [MR/K025023/1]. 
Competing interests None declared.

Provenance and peer review Not commissioned; externally peer reviewed.

Data sharing statement Data can be obtained from the corresponding author on request.

Open Access This is an Open Access article distributed in accordance with the terms of the Creative Commons Attribution (CC BY 4.0) license, which permits others to distribute, remix, adapt and build upon this work, for commercial use, provided the original work is properly cited. See: http:// creativecommons.org/licenses/by/4.0/

\section{REFERENCES}

1. Kohl HW III, Craig CL, Lambert EV, et al. The pandemic of physical inactivity: global action for public health. Lancet 2012;380:294-305.

2. Chastin SF, Scwarz U, Skelton DA. Development of a consensus taxonomy of sedentary behaviors (SIT): report of Delphi round 1. PLOS ONE 2013;8:e82313.

3. Sedentary Behaviour Research Network. Standardized use of the terms "sedentary" and "sedentary behaviours". Appl Physiol Nutr Metabol 2012;37:540-2.

4. Healy GN, Matthews CE, Dunstan DW, et al. Sedentary time and cardio-metabolic biomarkers in US adults: NHANES 2003-06. Eur Heart J 2011:32:590-7.

5. Harvey JA, Chastin SFM, Skelton DA. How sedentary are older people? A systematic review of the amount of sedentary behavior. $J$ Aging Phys Act 2015;23:471-87.

6. Proper KI, Singh AS, van Mechelen W, et al. Sedentary behaviors and health outcomes among adults: a systematic review of prospective studies. Am J Prev Med 2011;40:174-82.

7. Thorp AA, Owen N, Neuhaus M, et al. Sedentary behaviors and subsequent health outcomes in adults a systematic review of longitudinal studies, 1996-2011. Am J Prev Med 2011;41:207-15.

8. de Rezende LFM, Rey-López JP, Matsudo VKR, et al. Sedentary behavior and health outcomes among older adults: a systematic review. BMC Public Health 2014;14:333.

9. Dogra S, Stathokostas L. Sedentary behavior and physical activity are independent predictors of successful aging in middle-aged and older adults. J Aging Res 2012;2012:190654.

10. Department of Health. Start Active, Stay Active: a report on physical activity from the four home countries' Chief Medical Officers, 2011.

11. Guyatt G, Walter S, Norman G. Measuring change over time: assessing the usefulness of evaluative instruments. J Chronic Dis 1987;40:171-8

12. Kozey-Keadle S, Libertine $\mathrm{A}$, Lyden $\mathrm{K}$, et al. Validation of wearable monitors for assessing sedentary behavior. Med Sci Sports Exerc 2011;43:1561-7.

13. Healy GN, Clark BK, Winkler EAH, et al. Measurement of adults' sedentary time in population-based studies. Am J Prev Med 2011;41:216-27.

14. Bauman A, Ainsworth BE, Sallis JF, et al. The descriptive epidemiology of sitting: a 20 country comparison using the International Physical Activity Questionnaire (IPAQ). Am J Prev Med 2011:41:228-35.

15. Hekler EB, Buman MP, Haskell WL, et al. Reliability and validity of CHAMPS self-reported sedentary-vigorous intensity physical activity in older adults. J Phys Act Health 2012;9:225-36.

16. Chastin SFM, Culhane B, Dall PM. Questionnaire sitting time (IPAQ) compared to objective measurement via inclinometry (activPAL). Physiol Meas 2014;35:2319-28.

17. Kozey-Keadle S, Lyden K, Hickey A, et al. Validation of a previous day recall for measuring the location and purpose of active and sedentary behaviours compared to direct observation. Int J Behav Nutr Phys Act 2014;11:12.

18. Kmet LM, Lee RC, Cook LS. Standard quality assessment criteria for evaluating primary research papers from a variety of fields. Edmonton: Alberta Heritage Foundation for Medical Research, 2004;1-22.

19. Banks E, Jorm L, Rogers K, et al. Screen-time, obesity, ageing and disability: findings from 91266 participants in the 45 and Up Study. Public Health Nutr 2011;14:34-43.

20. Patel AV, Bernstein L, Deka A, et al. Leisure time spent sitting in relation to total mortality in a prospective cohort of US adults. Am J Epidemiol 2010;172:419-29.

21. Salmon J, Owen N, Crawford D, et al. Physical activity and sedentary behavior: a population-based study of barriers, enjoyment, and preference. Health Psychol 2003;22:178-88.
22. Gardiner PA, Healy GN, Eakin EG, et al. Associations between television viewing time and overall sitting time with the metabolic syndrome in older men and women: the Australian Diabetes Obesity and Lifestyle study. JAGS 2011;59:788-96.

23. Shields M, Tremblay MS. Sedentary behaviour and obesity. Health Rep 2008;19:19-30.

24. Katzmarzyk PT, Church TS, Craig CL, et al. Sitting time and mortality from all causes, cardiovascular disease, and cancer. Med Sci Sports Exerc 2009;41:998-1005.

25. Hamer M, Stamatakis E. Screen-based sedentary behaviour, physical activity, and muscle strength in the English Longitudinal Study of Ageing. PLOS ONE 2013;8:e66222.

26. Wareham NJ, Jakes RW, Rennie KL, et al. Validity and repeatability of the EPIC-Norfolk physical activity questionnaire. Int $J$ Epidemiol 2002;31:186-74.

27. Parsons TJ, Thomas C, Power C. Estimated activity patterns in British 45 year olds: cross-sectional findings from the 1958 British birth cohort. Eur J Clin Nutr 2009;63:978-85.

28. Armstrong T, Bull F. Development of the World Health Organization Global Physical Activity Questionnaire (GPAQ). J Public Health 2006; 14:66-70.

29. Stamatakis E, Davis M, Stathi A, et al. Associations between multiple indicators of objectively-measured and self-reported sedentary behaviour and cardiometabolic risk in older adults. Prev Med 2012;54:82-7.

30. Chau JY, Grunseit A, Midthjell K, et al. Sedentary behaviour and risk of mortality from all-causes and cardiometabolic diseases in adults: evidence from the HUNT3 population cohort. Br J Sports Med 2015;49:737-42.

31. Craig CL, Marshall AL, Sjöström M, et al. International Physical Activity Questionnaire: 12-country reliability and validity. Med Sci Sports Exerc 2003;35:1381-95.

32. Proper KI, Cerin E, Brown WJ, et al. Sitting time and socio-economic differences in overweight and obesity. Int J Obes 2007;31:169-76.

33. Visser M, Koster A. Development of a questionnaire to assess sedentary time in older persons-a comparative study using accelerometry. BMC Geriatr 2013;13:80.

34. Burazeri G, Goda A, Kark JD. Television viewing, leisure-time exercise and acute coronary syndrome in transitional Albania. Prev Med 2008;47:112-5.

35. Gardiner PA, Clark BK, Healy GN, et al. Measuring older adults' sedentary time: reliability, validity, and responsiveness. Med Sci Sports Exerc 2011;43:2127-33.

36. Ford ES. Combined television viewing and computer use and mortality from all-causes and diseases of the circulatory system among adults in the United States. BMC Public Health 2012;12:70.

37. Evenson KR, McGinn AP. Test-retest reliability of adult surveillance measures for physical activity and inactivity. Am J Prev Med 2005;28:470-8.

38. Wolf AM, Hunter DJ, Colditz GA, et al. Reproducibility and validity of a self-administered physical activity questionnaire. Int $J$ Epidemiol 1994;23:991-9.

39. Matthews CE, George SM, Moore SC, et al. Amount of time spent in sedentary behaviors and cause-specific mortality in US adults. Am J Clin Nutr 2012;95:437-45.

40. Salmon J, Bauman A, Crawford D, et al. The association between television viewing and overweight among Australian adults participating in varying levels of leisure-time physical activity. Int J Obes Relat Metab Disord 2000;24:600-6.

41. Washburn RA, McAuley E, Katula J, et al. The Physical Activity Scale for the Elderly (PASE): evidence for validity. J Clin Epidemiol 1999;52:643-51.

42. Clark BK, Winkler E, Healy GN, et al. Adults' past-day recall of sedentary time: reliability, validity and responsiveness. Med Sci Sports Exerc 2013;45:1198-207.

43. Clark BK, Pavey TG, Lim RF, et al. Past-day recall of sedentary time: validity of a self-reported measureof sedentary time in a university population. J Sci Med Sport 2016;19:237-41.

44. Balboa-Castillo T, León-Muñoz LM, Graciani A, et al. Longitudinal association of physical activity and sedentary behavior during leisure time with health-related quality of life in community-dwelling older adults. Health Qual Life Outcomes 2011;9:41-7.

45. Rosenberg DE, Norman GK, Wagner N, et al. Reliability and validity of the Sedentary Behaviour Questionnaire (SBQ) for adults. J Phys Act Health 2010;7:697-705.

46. Stamatakis E, Hamer M, Dunstan DW. Screen-based entertainment time, all-cause mortality, and cardiovascular events. J Am Coll Cardiol 2011;57:292-9.

47. Lynch BM, Friedenreich CM, Khandwala F, et al. Development and testing of a past year measure of sedentary behaviour: the SIT-Q. BMC Public Health 2014;14:899. 
48. Wijndaele K, de Bourdeaudhuij I, Godino JG, et al. Reliability and validity of a domain-specific last 7-d sedentary time questionnaire. Med Sci Sports Exerc 2014;46:1248-60.

49. Csizmadi I, Neilson HK, Kopciuk KA, et al. The Sedentary Time and Activity reporting Questionnaire (STAR-Q): reliability and validity against doubly labelled water and 7-day activity diaries. Am J Epidemiol 2014;80:424-35.

50. Mensah K, Maire A, Oppert JM, et al. Assessment of sedentary behaviours and transport-related activities by questionnaire: a validation study. BMC Public Heath 2016;16:753.

51. Dahl-Pederson IK, Hansen AW, Bjerregaard P, et al. Validity of the international physical activity questionnaire in the Arctic. Med Sci Sports Exerc 2013;45:728-36.

52. van Dyck D, Cardon G, Deforche B, et al. IPAQ interview version: convergent validity with accelerometers and comparison of physical activity and sedentary time levels with the self-administered version. J Sports Med Phys Fitness 2014;55:776-86.

53. Hagstromer M, Ainsworth BE, Sjostrom M. Comparison of a subjective and an objective measure of physical activity in a population sample. J Phys Act Health 2010;7:541-50.

54. Celis-Morales CA, Perez-Bravo F, Ibanez L, et al. Objective vs. self-reported physical activity and sedentary time: effects of measurement method on relationships with risk biomarkers. PLoS ONE 2012;7:e36345

55. Wanner M, Probst-Hensch N, Kriemler S, et al. Validation of the long international physical activity questionnaire: influence of age and language region. Prev Med Rep 2016;3:250-6.

56. Dyrstad SM, Hansen BH, Holme IM, et al. Comparison of self-reported versus accelerometer-measured physical activity. Med Sci Sports Exerc 2014;46:99-106.

57. Oyeyemi AL, Umar M, Oguche F, et al. Accelerometer-determined physical activity and its comparison with the International Physical
Activity Questionnaire in a sample of Nigerian adults. PLOS ONE 2014:9:e87233.

58. Hurtig-Wennlöf A, Hagströmer M, Olsson L. The International Physical Activity Questionnaire modified for the elderly: aspects of validity and feasibility. Public Health Nutr 2010;13:1847-54.

59. Grimm EK, Swartz AM, Hart T, et al. Comparison of the IPAQ-short form and accelerometry predictions of physical activity in older adults. J Aging Phys Act 2012;20:64-79.

60. Curry WB, Thompson JL. Comparability of Accelerometer- and IPAQ-derived physical activity and sedentary time in south Asian women: a cross-sectional study. Eur J Sport Sci 2015;15:655-62.

61. Alkahtani SA. Convergent validity: agreement between accelerometry and the Global Physical Activty Questionnaire in college-age Saudi men. BMC Res Notes 2016;9:436.

62. Gennuso KP, Matthews CE, Colbert LH. Reliability and validity of two self-report measures to assess sedentary behavior in older adults. J Phys Act Health 2015;12:727-32.

63. Busschaert C, de Bourdeaudhuij I, Van Holle V, et al. Reliability and validity of three questionnaires measuring context-specific sedentary behaviour and associated correlates in adolescents, adults, and older adults. Int J Behav Nutr Phys Act 2015;12:117.

64. Van Cauwenberg J, Van Holle V, De Bourdeaudhuij I, et al. Older adults' reporting of specific sedentary behaviours: validity and reliability. BMC Public Health 2014;14:734.

65. Beaton DE, Bombardier C, Katz JN, et al. A taxonomy for responsiveness. J Clin Epidemiol 2001:54:1204-71.

66. Gomersall SR, Pavey TG, Clark BK, et al. Validity of a self-report recall tool for estimating sedentary behaviour in adults. J Phys Act Health 2015;12:1485-91.

67. Hart TL, Swartz AM, Cashin SE, et al. How many days of monitoring predict physical activity and sedentary behaviour in older adults? Int J Behav Nutr Phys Act 2011;8:62. 